\title{
Traduire
}

Revue française de la traduction

$216 \mid 2008$

Question de confiance

\section{Masculin, féminin - Entre snobisme et parité}

\section{Françoise Fourault-Sicars}

\section{(2) OpenEdition \\ Journals}

Édition électronique

URL : http://journals.openedition.org/traduire/979

DOI : 10.4000/traduire.979

ISSN : 2272-9992

Éditeur

Société française des traducteurs

Édition imprimée

Date de publication : 1 mars 2008

Pagination : 45-58

ISSN : 0395-773X

\section{Référence électronique}

Françoise Fourault-Sicars, " Masculin, féminin - Entre snobisme et parité », Traduire [En ligne],

216 | 2008, mis en ligne le 01 mars 2008, consulté le 03 mai 2019. URL : http://

journals.openedition.org/traduire/979; DOI : 10.4000/traduire.979 


\section{Masculin, féminin - Entre snobisme et parité ${ }^{*}$}

\section{FranÇOISE Fourault-SicarS}

C'est en lisant des journaux dits féminins et d'autres dits plus sérieux, politiques ou économiques, que m'est venue l'idée de faire un petit exposé sur la féminisation dans la langue française. Au début, je me suis dit que ce serait simple et qu'il suffirait de faire un petit tableau en exposant les différentes règles et en donnant toutes les références utiles et les exemples trouvés.

Dès que j'ai commencé mes recherches, je me suis aperçue que le thème était en réalité très complexe et qu'il n'y avait pas de règles absolument précises ou que, s'il y en avait, personne ou presque ne s'en préoccupait. De plus, ces règles varient suivant les pays francophones. Pour faire court, et en référence au titre que j'ai donné à mon exposé, je dirai que le féminisme et la volonté de parité entre les hommes et les femmes, même si cette dernière est légitime et louable, conduisent souvent à des excès dans la féminisation des mots qui frisent le ridicule. Pour souligner jusqu'où peut aller l'excès (dans les deux sens) et les aberrations du langage, je commencerai par citer un exemple :

La France :

"Le capitaine Prieur est actuellement enceinte et l'accord prévoyait que dans ces circonstances, elle pouvait être rapatriée à Paris " (1988 affaire Greenpeace).

La Suisse :

Citation d'une association suisse dans ses statuts :

* Texte d'une communication présentée par l'auteur à la $14 \mathrm{e}$ rencontre du Réseau franco-allemand tenue à Nice, du 26 au 28 octobre 2007. 
"Pour des raisons de lisibilité, nous n'employons dans ce document que la forme féminine. Les hommes sont toutefois pris en considération au même titre que les femmes ".

Et sur le site allemand de cette même association, on trouve : 2006 : «Les statuts sont révisés. L'association souhaite, par cette révision, montrer la voie de l'égalité entre les sexes, et remplace tous les masculins par des féminins. "

Le problème de la féminisation dans le langage n'est pas nouveau. Je me contenterai d'aborder le sujet dans ses grandes lignes en passant les différents pays en revue ; étant de langue maternelle française, j'aborderai uniquement le sujet du point de vue de la francophonie, mais je tiens à signaler qu'il existe également des règles et conseils de rédaction non sexiste dans d'autres pays, notamment en Allemagne.

\section{Le Québec}

C'est au Québec que la féminisation de la langue française a pris son essor, dès la fin des années 1970 . Cette action correspond à la libération des femmes mais aussi à ce que l'on a appelé la « révolution tranquille " : elle coïncide également avec la défense du français sur un continent anglophone. L'Office de la langue française statue sur la francisation des termes; il a rendu plusieurs avis sur la féminisation des titres et sur l'affichage des postes. En 1986 est paru un document intitulé Titres et fonctions au féminin : essai d'orientation de l'usage, puis en 1991 : Au féminin; guide de féminisation des fonctions et des textes. De nombreuses industries et entreprises se sont dotées d'un guide à usage interne et encouragent leurs employés à suivre des cours de rédaction non sexiste.

C'est au Québec qu'on a commencé à former des féminins en -eure (ingénieure, professeure), suffixe refusé par l'Académie française en France. Il est dérivé du modèle un "prieur ", une " prieure " et jugé plus acceptable que -trice ou -euse ou -esse considérés comme péjora- 
tifs. Cette prise de position ne fait pas l'unanimité et certains demandent le retour aux règles traditionnelles de féminisation, ce qui semble conforme au souhait de l'Office dans son édition du Français au bureau datant de 1996. C'est aussi dans ce sens que vont les documents en français publiés par les différents gouvernements canadiens.

Louvrage de Céline Labrosse - Pour une grammaire non sexiste - va encore plus loin. Connue pour ses travaux sur le sexisme de la langue française, elle s'est donné pour but d'éradiquer le sexisme linguistique par la " dégenrisation " de la langue, c'est-à-dire de gommer l'association entre mots et sexe pour supprimer des marques de genre dans les mots. Elle propose également une réforme structurelle et remplace les terminaisons -és, -ées, par exemple dans aimés, aimées, par la terminaison en -ez, donnant alors aimez. Pour les terminaisons -is, -ies, $-u s$, -ues, nous aurions des tâches et des devoirs accompliz, des convives hindouz. Elle reprend également la proposition de la québécoise Françoise Marois, pour la création d'un pronom collectif mixte : illes. Nous n'écririons plus Ils sont allés en promenade ni Elle et il sont allés en promenade, mais Illes sont allez en promenade. Certaines de ses propositions ont été acceptées par deux syndicats.

\section{Le Luxembourg}

La création d'un ministère de la Promotion féminine en 1995 a permis au Luxembourg de se doter, entre autres, d'un Dictionnaire de la féminisation des noms de métiers, titres et fonctions (1998) bilingue allemand et français. La parité implique pour les Luxembourgeoises une féminisation lexicale et syntaxique. Cette féminisation s'aligne sur celle de la Suisse en ce qui concerne la féminisation syntaxique à quelques exceptions près (proviseur, réviseur). Les tirets (les citoyen-ne-s) sont utilisés dans les textes officiels, mais il ne semble pas y avoir de pratique systématique en matière de féminisation des textes. 


\section{La Belgique}

La Belgique a suivi les directives européennes et les textes officiels commencent à être " désexisés » dès 1988. En juin 1993, le Parlement de la Communauté française adopte un décret recommandant au secteur public sous sa tutelle de féminiser les noms de métier, fonction, grade ou titre dans tous les documents. Le Conseil supérieur de la langue française remet son avis le 5 juillet 1993 ; les règles qu'il préconise seront publiées dans le décret d'application de décembre 1993. Un guide, Mettre au féminin, est publié en 1994 par le Service de la langue française ; 42000 exemplaires en seront distribués. Voici quelques exemples de règles :

- pas de mots en -esse (vieillis) ;

- les noms masculins terminés par une consonne prennent en règle générale un -e final : agente, commise, échevine, principale ;

- avec parfois redoublement de la consonne finale (chirurgienne) ou apparition d'un accent grave : une préfete, une officière ;

- certains noms ne se féminisent pas : une écrivain, une chef, une conseil juridique, une médecin, une marin ;

- forme féminine également identique au masculin lorsqu'au nom ne correspond pas de verbe : une docteur, une ingénieur, une procureur, une professeur (sauf une ambassadrice, une chroniqueuse) ;

- les adjectifs et participes s'accordent systématiquement au féminin, même dans les appellations complexes, ce qui donne : une conseillère adjointe, mais aussi : une ingénieur technicienne... ;

- demandes d'emploi : forme féminine en entier, pas de "Cherchons un(e) mécanicien(ne) » ni "Cherchons mécanicien (H/F)";

- généralisation de l'emploi de Madame au lieu de Mademoiselle ;

- les dispositions québécoises et suisses en -eure, peuvent être éventuellement utilisées. "L'usage tranchera au cours des prochaines décennies ". 


\section{La Suisse romande}

Aujourd'hui, tous les cantons romands ont des bureaux ou ministères de l'Égalité des droits. Ils ont été à l'origine du Nouveau Dictionnaire féminin-masculin des professions, des titres et des fonctions. Par ailleurs, le Conseil fédéral s'est déclaré, en 1986, en faveur d'une rédaction administrative et législative rendant justice aux réalités sociales de notre temps. En juin 1991, la Chancellerie fédérale publiait un guide de Formulation non sexiste des actes législatifs et administratifs. Féministes et bureaux de l'égalité ont pris parti pour une féminisation systématique. Les formes féminines ont été favorisées pour que les femmes cessent d'être occultées de la société et de la langue.

La féminisation du vocabulaire paraissant une étape vers un langage égalitaire, on a voulu favoriser la désexisation du langage en introduisant des expressions telles que celles et ceux, toutes et tous, etc., et en recommandant de ne plus accorder systématiquement au masculin une suite de féminins et de masculins, mais a) de retourner à l'usage de l'accord au plus proche : les restaurateurs et restauratrices sont contentes de leur personnel -, b) de suivre l'ordre alphabétique : les papetières et les papetiers, mais les auditeurs et les auditrices, c) ou encore de mettre les verbes au pluriel lorsque les dénominations sont reliées par ou, ainsi : le chef ou la cheffe demandent...

L'emploi de parenthèses pour " inclure " le féminin a été déconseillé, car les parenthèses « désignent l'accessoire et peuvent rendre un texte illisible. " Et si l'emploi du trait d'union paraît symboliquement plus riche, son abus rendrait lui aussi les textes incompréhensibles. De même est déconseillé l'emploi de la barre oblique qui, d'une part, signifie l'alternance ou même l'exclusion en rhétorique et qui, d'autre part, rend les textes difficiles à lire ; il en va de même pour l'inclusion de majuscules (par exemple «touTEs" pour " tous et toutes" comme le fait l'allemand) qui semble incompatible avec les habitudes de lecture francophone et qui étonne hors des frontières helvétiques. C'est pourquoi on propose d'utiliser des termes génériques sachant toutefois qu'ils peuvent dépersonnaliser le texte et que l'emploi systématique de 
" on" reviendrait à " occulter les sexes". Toutes ces règles et conseils ont été publiés dans l'ouvrage Écrire les genres : guide d'aide à la rédaction administrative et législative épicène (1).

\section{La France}

En France, une Commission de terminologie pour la féminisation des métiers, titres et fonctions a été formée en 1984 par la ministre des Droits des femmes, Yvette Roudy. La Commission était composée de trente-deux linguistes, universitaires, enseignants, écrivains et représentants des divers ministères. Les décisions de la Commission ont été approuvées par le Commissariat général de la langue française et par le Comité consultatif de la langue française. Elles s'appuyaient également sur la loi 75-1349 du 31 décembre 1975 relative à l'emploi de la langue française, sur le décret 83-243 du 25 mars 1983 relatif à l'enrichissement de la langue française et sur la loi 83-635 relative à l'égalité professionnelle entre hommes et femmes. Les travaux de la Commission ont abouti à une circulaire relative à la féminisation des noms de métier, fonction, grade ou titre, publiée au Journal officiel du 11 mars 1986.

Le 17 décembre 1997, le président de la République, Jacques Chirac, et le Premier ministre, Lionel Jospin, ont réactivé cette circulaire déclarant que les textes administratifs devraient désormais faire usage des féminins pour les métiers ou fonctions ou titres des femmes. Cette décision a été approuvée le 14 mai 1998 par l'Assemblée nationale et, le 9 juillet de la même année, le ministère de l'Éducation publique la mettait en œuvre. Puis, en 1999, paraissait le guide d'aide à la féminisation des noms de métiers, titres, grades et fonctions portant le beau titre : Femme, j'écris ton nom(2) publié par le CNRS et l'Inalf et préfacé par Lionel Jospin.

(1) http://w3.jura.ch/communiq/02-2002c.htm

(2) http://www.ladocumentationfrancaise.fr/rapports-publics/994001174/index.shtml 
Après la première tentative du gouvernement en 1984 en faveur de la féminisation des titres et fonctions et, d'une manière générale, du vocabulaire concernant les activités des femmes, l'Académie française fait publier une déclaration rappelant le rôle des genres grammaticaux qui conclut : "En français, la marque du féminin ne sert qu'accessoirement à rendre la distinction entre mâle et femelle. Tous les emplois du genre grammatical constituent un réseau complexe où la désignation contrastée des sexes ne joue qu'un rôle mineur. Des changements, faits de propos délibéré dans un secteur peuvent avoir sur les autres des répercussions insoupçonnées ». Cet avertissement n’a pas été entendu et le guide précité, publié en 1998, a été perçu par la presse plus ou moins comme une directive légale, alors que la Commission générale de terminologie venait de remettre un rapport déconseillant la féminisation des titres, grades et fonctions par distinction avec les noms de métiers, où le féminin découle de l'usage même. On n'en a pas tenu compte non plus (voir les liens pour consulter le texte de L. Jospin et celui de l'Académie).

\section{Avis de l'Académie française :}

\section{1) Le genre masculin}

En français, le genre masculin a une valeur collective et générique. Il est inutile, pour désigner un groupe de personnes composé d'hommes et de femmes, de répéter le même substantif ou le même pronom au féminin puis au masculin "les électrices et les électeurs", toutes celles et tous ceux, etc., formules qui ne disent rien de plus que les électeurs, tous ceux, etc. On évitera également d'indiquer entre parenthèses ou après une barre oblique la marque du féminin les animateurs/trices, les adhérent(e)s. Ces redondances gênent la lecture et alourdissent le style.

\section{2) Les néologismes ou aberrations lexicales}

Les seuls féminins français en -eure (ex. supérieure) sont ceux qui proviennent de comparatifs latins en -or. Éviter donc les néologismes 
comme : docteure, proviseure, procureure, rapporteure, réviseure, auteure, etc.

Certains sont absurdes, car le féminin existe : instituteure pour institutrice, chercheure pour chercheuse, etc.

Éviter aussi les néologismes tels que agente, cheffe, maîtresse de conférences, écrivaine, etc.

Seul le genre masculin, qui est le genre non marqué car il a la capacité de représenter les éléments relevant des deux genres, peut traduire la nature indifférenciée des titres, grades, dignités et fonctions.

L'Académie souligne que l'instauration d'une réelle égalité entre les hommes et les femmes dans la vie politique et économique rend indispensable la préservation de dénominations collectives et neutres, donc le maintien du genre non marqué chaque fois que l'usage le permet. Le choix systématique et irréfléchi de formes féminisées établit au contraire, à l'intérieur même de la langue, une ségrégation qui va à l'encontre du but recherché.

\section{Avis plus nuancé}

Je recommande vivement la lecture de Femme, jécris ton nom(3), un rapport très complet, qui résume bien les tenants et les aboutissants (ou non-aboutissants) de ce problème, avec un historique, les règles de féminisation des titres, grades et fonctions, les mots empruntés aux langues étrangères, les exceptions, les mots composés. Il aborde les difficultés et les objections : homonymie (ex. moissonneuse, balayeuse, médecine), euphonie (sapeuse-pompière), dévalorisation (ambassadrice, pharmacienne $=$ la femme de.../camelot, camelote). Il aborde le rôle de l'adjectif et donne une liste des fonctions, titres et métiers, dont certains sont vraiment drôles. À l'encontre des positions prises par l'Académie, ce rapport affirme qu'il n'y a pas de forme neutre en français mais seulement deux genres, le féminin et le masculin, ainsi que des

(3) Voir supra. 
termes dits épicènes, à savoir identiques pour les deux sexes (un monstre, une victime, etc.).

Règle du masculin : le masculin a bien une certaine généricité au pluriel, mais c'est le singulier qui pose problème. On ne doit l'utiliser comme générique que lorsque l'on parle d'un individu en général.

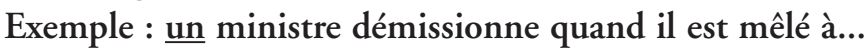

Quand on vise une personne précise, il convient d'adopter le genre qu'implique son sexe. Exemple : Madame X est la ministre de la Culture.

Règle générale à appliquer : garder la richesse de la langue et l'opposition du générique et du spécifique. Exemple : «Dans cet hôpital, les fonctions de chirurgien (générique) sont occupées par une chirurgienne (spécifique)». C'est une erreur de désigner une personne singulière par un masculin générique. Dire «Mme le ministre » ou « mon avocat ", c'est nier la singularité de la personne. "Madame le ministre est contraire à la logique (générique dans un énoncé spécifique), à la grammaire et à la civilité (elle impose un masculin à une personne féminine particulière »).

\section{Les termes du débat : pour et contre}

En France surtout, la féminisation rencontre des oppositions de principe.

a) Beaucoup de femmes tiennent encore au titre masculin (Mme X, avocat au barreau de..., député de...) ; mais cette réserve devient rare.

b) Le masculin, "genre non marqué ", vaut pour les deux sexes ; c'est exact quand le terme, pluriel ou singulier, est pris dans son sens général : "les clients - les voisins - les Anglais... ; le client du commerçant... ", mais cela n'a jamais empêché la création spontanée de féminins quand une fonction cessait d'être réservée aux hommes.

c) C'est pourtant la motivation de l'opposition catégorique de l'Académie qui, dans la 9e édition de son Dictionnaire, en cours de réalisation, n'accepte que le masculin pour ambassadeur (sauf au figuré ou épouse), conseiller (sauf privé), député, juge, magistrat, maire, ministre, etc. 
d) Le gouvernement, lui, féminise le titre de ses ministres et des députés ; le Journal officiel et la Documentation française connaissent des professeure, procureure, consule, etc. Plusieurs de ces féminins deviennent courants (au Québec plus qu'en France) dans la presse et dans l'usage. Il semble donc qu'une certaine distance ait été prise par rapport aux propos tenus en 1955 par le grammairien Albert Dauzat :

"La femme qui préfere pour le nom de sa profession le masculin au féminin accuse par là même un complexe d'infériorité qui contredit des revendications légitimes. Dérober son sexe derrière le genre adverse, c'est le trahir. [Dire] madame le docteur, c'est reconnaître implicitement la supériorité du mâle, dont le masculin est l'expression grammaticale. "

\section{Les usages}

Dans la fonction publique, les pratiques different :

- Grands corps et hautes juridictions sont opposés à la féminisation ;

- Dans les métiers, la féminisation est courante (exemple : directrice d'école) et partout sauf là où les termes n'ont pas de forme féminine évidente (contrôleur financier, trésorier, etc.) ;

- Dans la justice, même séparation dans les métiers (greffière, directrice, etc.) et aucune féminisation directe chez les notaires, huissiers de justice, etc. L'usage courant parle pourtant de la juge, la magistrate, mais l'usage du masculin reste prédominant, exemple : Madame le procureur etc. ;

- Dans le monde diplomatique, usage traditionnel, pas de féminisation des titres ;

- Dans l'armée (sauf dans la marine) l'usage est de dire « mon commandant, mon général " mon étant l'abréviation de monsieur. Pour les femmes, la pratique a gardé les titres masculins et supprimé le " mon" (pour garder, dit-on, la neutralité des appellations, quel que soit le sexe). Les titres militaires féminins existent : amirale, générale, lieutenante, colonelle, etc. sauf pour le mot « officier»; 
- Usages sociaux : Mme Émile Dupont devient de plus en plus Mme Françoise Dupont. On peut écrire aussi Madame et Monsieur Dupont.

Dans la langue courante, certains termes se sont répandus facilement, comme la chef, l'écrivaine ou la magistrate. Dans d'autres cas, on hésite à utiliser la forme féminine, comme déjà évoqué : assureuse, pompière, difficile ou souvent dépréciatrice (exemple : couturier, métier noble, couturière, au bas de l'échelle) et on préfere marquer le féminin par l'article : une chef, une professeur (forme épicène) ou apposer le mot femme - exemple : une femme médecin.

La présence croissante des femmes dans toutes les professions et l'influence des médias modifient les traditions et accélèrent la féminisation. Dans l'administration et l'Éducation nationale, le gouvernement fait toujours référence au guide Femme, j'écris ton nom et recommande de l'appliquer, ce qui n'est fait que rarement, vu les polémiques soulevées chez les Français quand on touche à leur langue.

\section{Féminisation : de nombreux points communs}

Même s'il existe quelques différences de pays à pays dans la manière de féminiser, les points communs sont plus nombreux que les divergences. Celles-ci sont d'ailleurs, avant tout, le signe de la richesse et de la multiplicité des pratiques. Il ne saurait y avoir une féminisation standard, pas plus qu' un français standard, sans appauvrissement de la langue, en vertu du principe selon lequel chaque culture (ré)invente et nourrit la francophonie.

Tout le monde ou presque s'accorde pour adopter un pluriel générique au masculin. Tous les guides de féminisation sont d'accord sur le fait de mettre un article en accord avec le sexe de la personne exerçant le métier ou la fonction. On dira ainsi, selon les pays, une commis ou une commise, une procureur ou une procureure ou encore une procureuse. La plupart des dénominations de métiers, des fonctions ou des titres sont identiques dans tous les pays. On parle partout de la juge, la ministre comme de la suppléante, d'une ambassadrice, d'une acheteuse, 
d'une marchande ou d'une joueuse de tennis. Pourtant, si certaines des règles de féminisation sont les mêmes dans les manuels de grammaire, les usages continuent à différer d'un pays à un autre. Les différences sociales et politiques se reflètent dans la diversité des usages, de sorte qu'on trouvera pour le féminin de chef, les variantes une chef, une chèfe ou une cheffe, par exemple, selon les pays. La féminisation lexicale est néanmoins, dans l'ensemble, très harmonisée.

Le débat est plus vif sur la nécessité de désexisation des textes ou sur la féminisation syntaxique (c'est-à-dire de l'accord grammatical). Pour le moment, ce sont les pays qui ont été les premiers à introduire une féminisation lexicale qui se sont engagés dans cette autre voie avantgardiste, à savoir le Québec et la Suisse. L'avenir seul nous dira si elle s'imposera ou non.

\section{Annexe}

Petits exemples pour rire :

"La règle générale de formation du féminin (ajouter un " $\mathrm{e}$ " au masculin - comme dans rouquin/rouquine) est battue en brèche par Fasolat \& Lyant ${ }^{(4)}$ :

un loup / une loupe un pont / une ponte un chais / une chaise un frais / une fraise un gland / une glande un vent / une vente un port / une porte un pair / une paire un patin / une patine un lézard vert / une lézarde verte... »

(4) P. Fasola et J.-Ch. Lyant : Grammaire turbulente du français contemporain, Paris, Seghers, 1989. 
Traductrice, Françoise Fourault-Sicars a toujours cultivé deux passions : l'allemand et l'art. Titulaire du Diplôme national (Section Textiles et décoration) de l'École des Beaux-Arts d'Angers, elle a enseigné la théorie de la couleur et animé des ateliers artistiques pour enfants et enseigné le dessin en lycée, tout en continuant à cultiver sa passion de l'Allemagne et de sa langue. Une rencontre faite dans le cadre du jumelage de la ville d'Angers marque un tournant décisif dans sa vie et l'amène à s'installer pour 21 ans à Osnabrück, où elle cumule expositions artistiques, activité d'enseignement et de commerce... et vie conjugale, avant d'être embauchée comme traductrice-interprète par un grand fabricant de meubles. Elle poursuit parallèlement des études de traduction qui lui permettront d'obtenir un diplôme national et d'être traductrice assermentée auprès des tribunaux d'Osnabrïck, avant de revenir s'installer dans le Sud de la France où elle poursuit jusqu'à ce jour son activité de traductrice.

www.fourault-sicars.de

\section{Bibliographie :}

Femme, j'écris ton nom - Guide d'aide à la féminisation (119 pages) 1999, préface de Lionel Jospin

www.culture.gouv.fr./culture/dglf/cogeter/feminisation/preface.pm. guide.fem.html

Mettre au féminin - Guide de féminisation des noms de métier, fonction, grade ou titre. 2e édition. Bruxelles, 2005. 80 pages. Voir http://www2.cfwb.be/franca/femini/feminin.htm

Pour trouver l'équivalent féminin d'un masculin donné avec lien vers la règle applicable voir le site de l'ATILF (Analyse et traitement informatique de la langue française) CNRS - Nancy :

http://atilf.atilf.fr/gsouvay/scripts/feminin.exe

Appellations professionnelles - Note de l'Éducation nationale de mars 2000 :

www.education.gouv.fr/botexte/bo000309/MEND0000585X.htm 
Égalité des sexes et développement Concepts et terminologie : http://cifdi.francophonie.org/genre/genre5.htm

Femmes de l'Équipement/Féminisation des noms de métier - Rappel des règles (ministère français de l'Écologie, du Développement et de l'Aménagement durables 2004) :

www.equipement.gouv.fr/article.php3?id_article=205

Guide de formulation non sexiste (Suisse), disponible en français et en allemand sur le site :

www.admin.ch/ch/f/bk/sp/doc/guide-de-formulation-non-sexiste.html

«Le féminin entre crochets » Entretien avec Benoîte Groult : www.regards.fr/article/ ?id=344

Organisation féministe des Chiennes de garde - Articles sur le sexisme dans le langage / Fiches de lecture - Les mots et les femmes : http://chiennes de garde.org/rubrique.php3 ?id_rubrique $=8$ http://chiennesdegarde.org/article.php3?id_article= 51 http://chiennesdegarde.org/article.php3?id_article=52 